\title{
B.1.617.2 variant and increasing surge of COVID-19 in Nepal
}

\author{
Umid Kumar Shrestha* \\ Nepal Mediciti Hospital, Bhaisepati, Lalitpur, Nepal
}

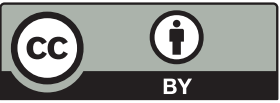

This work is licensed under a Creative Commons Attribution 4.0 Unported License.

\begin{abstract}
As the coronavirus disease 2019 (COVID-19) pandemic goes on, there has been an evolution of severe acute respiratory syndrome corona virus 2 (SARS-CoV-2) leading to the mutations. As of May 2021, the B.1.617 variant has become the dominant strain across India and has spread to about 40 nations. Among different strains of B. 1.617, the mutations of great concern are E484Q, L452R, P681R and T478K, because of the stronger affinity of the spike protein of these mutants for the Angiotensin Converting Enzyme (ACE) 2 receptor making it more transmissible and infectious, and causing decreased recognition capability of the immune system. Among the sub-variants of B.1.617, the B.1.617.3 shares the L452R and E484Q mutations found in B.1.617.1, whereas B.1.617.2 does not have the mutation E484Q, but it has the T478K mutation, not found in B.1.617.1 and B.1.617.3. With the increasing surge of COVID-19 in India with dominant B.1.617.2 variant, Nepal also witnessed the parallel increasing number of COVID-19 cases starting from the early weeks of April 2021, which could have been triggered by the influx of people due to the long open border of Nepal with neighboring India. Obviously, as of May 2021, the second wave of COVID-19 in Nepal has been linked with this new variant B.1.617.2 of SARS-CoV-2. Despite the challenges imposed by the new variant, the vaccines are likely to remain effective against different variants including B.1.617.2 and to limit severe disease. Moreover, there is an urgent need of an effective locally based system for the testing, contact tracing, and isolation. The risk reduction behavior, such as washing hands frequently, keeping at least $1 \mathrm{~m}$ distance from others and wearing a mask, should be adapted to the daily habits during this COVID-19 pandemic era. There should be a long term planning for the control of COVID-19 with the effective vaccination and public health measures; otherwise, even after the control of the current surge, the next wave of COVID-19 might be knocking at door in the coming days.
\end{abstract}

\section{KEY WORDS}

COVID-19; mutation; variant; B. 1.617.2; Nepal

As the coronavirus disease 2019 (COVID-19) pandemic goes on, there has been an evolution of severe acute respiratory syndrome corona virus 2 (SARS-CoV-2) leading to the mutations. The virus with one or more new mutations is referred to as a "variant" of the original virus. A number of naturally selected mutations in the spike protein of SARS-CoV-2 in several variants have been shown to affect infectivity, human-to-human transmission, pathogenesis and immune escape. ${ }^{?}$
Earlier in 2020, Nepal reported the variant arising from Wuhan (China), but in January 2021, Nepal witnessed cases of UK variant B.1.1.7 in three people returning from UK, resulting in the fear that Nepal might get overwhelming number of UK variant very soon; however, later on, Nepal reported the increasing surge of COVID-19

*Corresponding Author

Umid Kumar Shrestha, MD, PhD

Nepal Mediciti Hospital, Bhaisepati, Lalitpur, Nepal

Email: umidshrestha@gmail,com 
after second week of April 2021, which was thought to be originated from the so-called "Indian variant". This later variant of SARS CoV-2, more accurately called B.1.617, is a coronavirus variant that has played a big role in the second wave of infections in India. ${ }^{2}$ The arrangement of the mutated spike protein as revealed by the mapping of key mutations on the furin-cleaved crystal structure of SARSCoV-2 spike glycoprotein in complex with Angiotensin Converting Enzyme (ACE) 2 receptor is shown in figure 1.

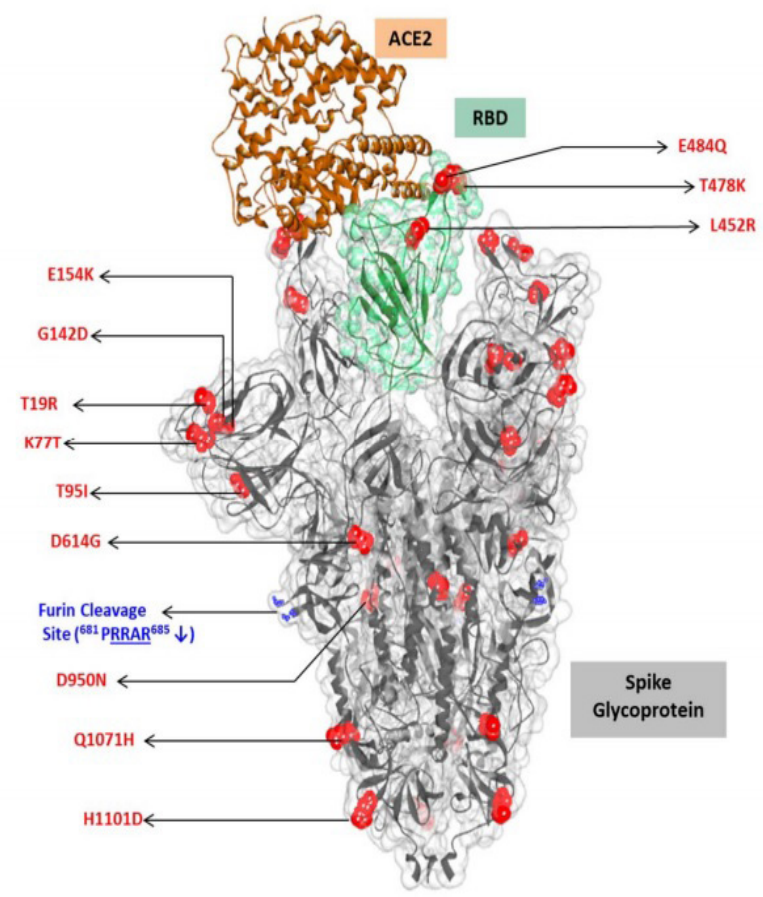

Figure: Mapping of key mutations on the furin-cleaved crystal structure of SARS-CoV-2 spike glycoprotein (grey surface view) in complex with Angiotensin Converting Enzyme (ACE) 2 receptor (brown solid ribbon). Receptor Binding Domain (RBD) region shown in green ${ }^{3}$

As of May 2021, the B.1.617 variant has become the dominant strain across India and has spread to about 40 nations. $^{2}$ The sub-variants of B.1.617 has been categorised into B.1.617.1, B.1.617.2, and B.1.617.3. The mutations observed in spike protein are as follows 4 :

B.1.617.1: (T95I), G142D, E154K, L452R, E484Q, D614G, P681R, Q1071H

B.1.617.2: T19R, (G142D), 156del, 157del, R158G, L452R, T478K, D614G, P681R, D950N

B.1.617.3: T19R, G142D, L452R, E484Q, D614G, P681R, D950N
Among them, the mutations of great concern are E484Q, L452R, P681R and T478K, because of the stronger affinity of the spike protein of these mutants for the ACE2 receptor making it making it more transmissible and infectious, and causing decreased recognition capability of the immune system.

Among these sub-variants, B. 1.617.3 shares the L452R and E484Q mutations found in B.1.617.1, whereas B.1.617.2 does not have the mutation $\mathrm{E} 484 \mathrm{Q}$, but it has the T478K mutation, not found in B.1.617.1 and B.1.617.3.

The variant B.1.617.3 was first detected in India in October 2020, followed by B.1.617.1 and B.1.617.2 in December

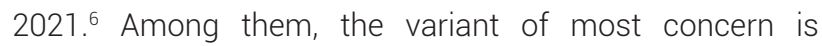
B.1.617.2. It remained rare in India until early March 2021, when it became the dominant variant reported. It seems to have spread to many other countries, including Nepal and is increasing rapidly. On 7 May 2021, B.1.617.2 was reclassified by Public Health England to a Variant Of Concern (VoC) due to evidence that it is at least as transmissible as B.1.1.7 (UK variant). ${ }^{7}$ On 11 May 2021, the World Health Organization also classified B.1.617.2 as a VoC. ${ }^{8,9}$ There are still a lot of things that we do not know about the B.1.617.2 variant. It seems that the dominant B.1.617.2 variant is very likely to be more transmissible. ${ }^{3}$

With the increasing surge of COVID-19 in India with dominant B.1.617.2 variant, Nepal also witnessed the parallel increasing number of COVID-19 cases starting from the second weeks of April 2021, which could have been triggered by the influx of people from India due to the long open border of Nepal with neighboring India. Obviously, as of May 2021, the second wave of COVID-19 in Nepal was presumed to be due to the dominant new variant B.1.617.2 of SARS-CoV-2.

In Nepal, according to the result of whole-genome sequencing (WGS) of 35 samples during that period, B.1.617.2 variant was detected in 97 per cent samples while the B.1.617.1 variant was reported from other 3 per cent samples. ${ }^{10}$ The sequencing was carried out at CSIR Institute of Genomics and Integrative Biology in India, a WHO-recognized center of excellence in genomic sequencing. ${ }^{10}$ Though the sample size of gene sequencing was very small in that study, B.1.617.2 variant can still be presumed to be the dominant variant, which might have already shown its possible effect of high transmissibility, infectivity and evasion of the immunity during the second wave of COVID-19 in Nepal.

As of May 2021, Nepal has reported the presence of total four strains of SARS-CO-V-2, the first one is of original 
Wuhan (China) strain, the second one is of UK variant (B.1.1.7) and the thrid and fourth ones being the Indian variant (B.1.617.1 and B.1.617.2).

The sudden increase in the surge of COVID-19 in the second wave of COVID-19 pandemic in Nepal after the second week of April 2021 is shown in the figure $2 .{ }^{11}$

The increasing number of hospitalization and death in the second wave suggests that the B.1.617.2 variant might be causing more severe disease. With the existence of prolonged COVID-19 pandemic, there might be a possibility of a 'pandemic fatigue' among the people with decreasing adherence to risk reduction strategies of wearing mask, hand washing and social distancing. "Lockdown" has been used as a tool to break the chain of the transmissibility of the virus, but if the surge of COVID-19 continues, and lockdowns gets extended, or get more restrictive, the people have to pay a heavy price both in terms of life and livelihood.

Despite the challenges imposed by the new variant, the vaccines are likely to remain effective against different variants including B.1.617.2 and to limit severe disease. Hence, the only solution to fight against COVID-19 at the current situation is to vaccinate, vaccinate and vaccinate.

\section{Active Cases}

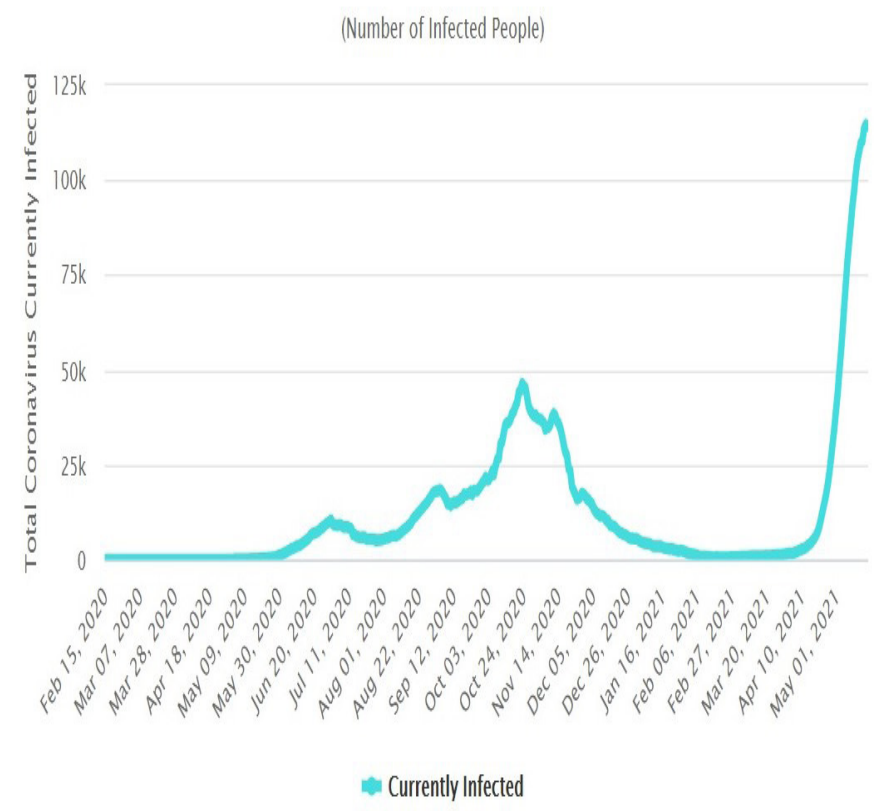

Figure 2: Active case of COVID-19 in Nepal showing the sudden increase in the surge after the second week of April 2021.
The vaccination will help to curtail the spread of infection to some extent and save fatalities to a great extent. However, the progress in vaccinations needs to be geared up significantly if we are to win the battle against COVID-19. Moreover, there is an urgent need of an effective locally based system for the testing, contact tracing, and isolation; for an effective isolation, there should be an enhanced financial and practical support to the needy people so that they can self-isolate effectively. The risk reduction behavior, such as washing hands frequently, keeping at least $1 \mathrm{~m}$ distance from others and wearing a mask, should be adapted to the daily habits during this COVID-19 pandemic era. There should be a long term planning for the control of COVID-19 with the effective vaccination and public health measures; otherwise, even after the control of the current surge, the next third wave of COVID-19 might be knocking at door in the coming days.

May 20, 2021

\section{CONFLICT OF INTEREST: None}

\section{REFERENCES:}

1. Wan Y, Shang J, Graham R, et al. Receptor Recognition by the Novel Coronavirus from Wuhan: an Analysis Based on Decade-Long Structural Studies of SARS Coronavirus. Gallagher T, editor. J Virol. 2020; 94(7):e00127-20, /jvi/94/7/ JVI.00127-20.atom.

2. Available from the internet [https://www.nature.com/ articles/d41586-021-01274-7]

3. Cherian S, Potdar V, Jadhav S, et al . Convergent evolution of SARS-CoV-2 spike mutations, L452R, E484Q and P681R, in the second wave of COVID-19 in Maharashtra, India. bioRxiv preprint doi: https://doi.org/10.1101/2021.04.22.440932

4. SARS-CoV-2 Variant Classifications and Definitions. Centers for Disease Control and Prevention. 12 May 2021. Available from the internet [https://www. cdc.gov/coronavirus/2019-ncov/cases-updates/variantsurveillance/variant-info.html]

5. Giacomo SD, Mercatelli D, Rakhimov A, et al. Preliminary report on severe acute respiratory syndrome coronavirus 2 (SARS-CoV-2) Spike mutation T478K. Journal of Medical Virology 2021 May 5. doi:10.1002/ jmv.27062. PMID 33951211.

6. Weekly epidemiological update on COVID-19 - 27 April 
B.1.617.2 variant and increasing surge of COVID-19 in Nepal | Editorial

2021. World Health Organization. 27 April 2021.

7. Confirmed cases of COVID-19 variants identified in UK.15 April 2021. Available from the internet [https://www.gov.uk/ government]

8. WHO labels a Covid strain in India as a 'variant of concern' - here's what we know, CNBC, 11 May 2021

9. WHO says India Covid variant of 'global concern', BBC News, 11 May 2021

10. COVID-19 variant in Nepal. Ministry of Health and Population, Government of Nepal. 18 May, 2021

11. Available from the internet [https://www.worldometers. info/coronavirus/country/nepal/] 